\title{
Recognizing the Danger Signs and Health Seeking Behaviour of Mothers in Childhood Illness in Karachi, Pakistan
}

\author{
Anwar-ul-Haq ${ }^{1}$, Hameed Mumtaz Durrani ${ }^{2, *}$, Ramesh Kumar ${ }^{3}$, Salma Mumtaz Durrani ${ }^{4}$ \\ ${ }^{1}$ Airport Health Dept. Karachi ,Ministry of National Health Services Regulation \& Coordination Government of Pakistan \\ ${ }^{2}$ Department of Public Health \& Community Medicine, AJK Medical College - University of Azad Jammu \& Kashmir, Pakistan \\ ${ }^{3}$ Health Services Academy, Pakistan \\ ${ }^{4}$ Alumni - Rawalpindi Medical College, Pakistan
}

Copyright (C) 2015 Horizon Research Publishing All rights reserved.

\begin{abstract}
Introduction: Maternal behaviour regarding child health care has been recognized as an important social and anthropological factor explaining high mortality rates among children under five years of age. Delays in seeking appropriate health care is known to be the largest risk behaviour which contribute large number of child deaths in developing countries especially in Pakistan. Mothers are mostly responsible for their child health, however, the importance of knowledge among mothers while in identifying the danger sign in their child are very imperative for preventing their illness. Method: A cross sectional study was conducted in an urban setting in Karachi city. The objective of study was to assess the knowledge of mother's regarding danger signs in their child illness and their care seeking behavior with aims to improve the neonatal and child health in Pakistan. Results: The majority of respondent mothers $(81.5 \%)$ were literate, housewives $(90 \%)$. Regarding child care the mothers are responsible for the immediate needs of their child. In the context of breast feeding and immunization of the infants, majority of the mothers were aware of practicing early initiation and uses of colostrum because of its importance. Although a large proportion of mothers $(90.1 \%)$ sought 'appropriate' care for childhood illness, generally care was sought from private doctors (69.4\%) instead of government medical practitioners. Conclusion: Study concluded that the Health seeking behviour of mothers has been found appropriate, however, their knowledge regarding the danger signs and illness of their children was reported low. It is recommended that some urgent awareness intervention by the policy makers for reducing the child death should be given to increase their knowledge on the danger sign of child diseases in Pakistan.
\end{abstract}

Keywords Child Health Care, Maternal Behaviour, Childhood Illness

\section{Introduction}

Maternal knowledge has positive impact on their health seeking behaviour and also for their child health in the many countries. However, health education for mothers have played a pivotal role in prevention of illnesses among the children.. In developing countries every year 12 million children die before they reach their fifth birthday, out of them many die during their first year of life. Seven out of ten of these deaths are due to acute respiratory tract infections (mostly pneumonia), diarrhea, measles, malaria or malnutrition - or a combination of which all can easily be prevented through changing the behaviour of women through health education on their child illness. According to the World Health Organization (WHO), over 4 billion acute cases of diarrheal diseases occur every year, primarily in children in developing countries. Two million children die annually from diarrhea. Lower respiratory tract infections such as pneumonia are another scourge for children and almost 4 million people died from lower respiratory infections in 2002, many of them children under five. ${ }^{1}$

Child deaths are not evenly distributed over the $0-5$ year period. The youngest are most vulnerable, $69 \%$ of under-five deaths occur in infancy (the first year of life), particularly during the neonatal period (the first month of life). But there are striking differences between all the six regions of world as per the WHO classification. . Most child deaths occur in low and middle-income countries and Pakistan is one of them where the number child death is very high and comes under the top ten countries of the world. Close to half in the WHO African Region and more than one quarter in the WHO South-East Asia Region. Moreover, the decreasing trends in the level of mortality vary widely between regions and countries, and progress towards the MDG 4 has been uneven. For example, the WHO African Region observed the smallest reductions in the past decades, while progress continued to accelerate in the WHO Region of the Americas 
and in the European Region. ${ }^{2}$

Countries in South-East Asia Region (SEAR) contribute to nearly $40 \%$ of these deaths. More than nine out often of these deaths occur in six countries of the region, namely Bangladesh, Bhutan, India, Indonesia, Myanmar and Nepal. ${ }^{3}$ In Pakistan, the childhood mortality has declined in the past 20 years but has remained relatively constant since 2003 . Pakistan is experiencing high childhood mortality and morbidity. Every year 700,000 children die and most of them of very common problems like measles, malnutrition, ARI, diarrhea and other vaccine preventable diseases. Currently, one in every 11 children in Pakistan dies before his or her fifth birthday. The Infant mortality rate is 78/1000 (2002-06) and under five mortality rate is $94 / 1000$ live births. Children living in rural areas are at higher risk for death than those living in urban areas. (PDHS 2006-07). ${ }^{4}$ Nearly half (43\%) of the population of Pakistan comprises of children 15 years of age who account for $60 \%$ of the national morbidity. In Pakistan, despite improving trends in mortality rates, ARI causes deaths of $13 \%$ and diarrhea causes deaths of $11 \%$ of children under 5 years ${ }^{4}$.

Responsiveness, a mother's/caregiver's prompt, contingent and appropriate interaction with the child, is a vital parenting tool with wide-ranging benefits for the child, from better cognitive and psychosocial development to protection from disease and mortality. Interventions are effective in enhancing maternal responsiveness, resulting in better child health and development, especially for the neediest populations. Since these interventions were feasible even in poor settings, they have great potential in helping to achieve the MDG-4. ${ }^{5}$

Where the quality of care at health facilities is adequate, care-seeking interventions have the potential to substantially reduce mortality. This is illustrated by the large number of children who die in developing countries without ever reaching a health facility, and amongst those who are taken to the health facility but then die, many deaths are attributed to delays in seeking care. Appropriate care-seeking is of particular importance in areas where access to health services is limited, because it is in these areas that caregivers would benefit most from being able to discern which episodes require care at a health facility, and which can be successfully treated at home. ${ }^{6}$

In Pakistan, The government has introduced WHO guidelines for acute respiratory infections (ARI) nationwide after training doctors and community health workers to use the technique. Use of these guidelines to improve the diagnosis and treatment of ARI among hospital outpatients under the age of five has halved pneumonia death rates among children admitted to hospital, reduced the inappropriate use of antibiotics, and led to major savings in health care costs. A study on the impact of the new treatment guidelines at the Islamabad Children's Hospital revealed that death rates among children admitted to hospital with severe pneumonia fell from about $10 \%$ to about $5 \%$ in a two year period. $^{7}$
Women's status is not homogenous in terms of individual and community traits, mobility, decision-making, and access to resources, economic autonomy and the relationship and communication with husband or elder of the family. In addition to food, sanitation, and facilities, children need adequate care at home for survival and optimum development. The World Health Organization estimates that seeking prompt and appropriate care by care givers could reduce child deaths by $20 \%$. Adequate recognition of danger signs is a vital parenting tool required wide ranging benefits for the child development and protection from morbidities and mortalities.

This study explores the knowledge of mothers regarding the recognition of danger signs in childhood illness and the types of care seeking they used, so that gaps in knowledge can be identified and used to help the policy makers to set strategies or interventions for timely care for children so that morbidity and mortality due to common childhood illnesses is reduced.

\section{Methodology}

A cross sectional study was conducted at the Shah Faisal Town, Karachi. The mothers who have child under 5 years of age with history of illness like diarrhea and/or ARI during the last 15 days were included in the study, while those mothers whose children had any other infectious diseases and ARI or ADD before 15 days, were excluded from the study. Multistage random sampling technique was done for data collection purpose. During the first stage sampling, a list of LHW was obtained from the Town Health Office; and 10 lady health workers were randomly selected. During the second stage, first house from the community was selected randomly, and then subsequent houses were selected by using systematic random sampling. Finally, the women with child under 5 years of age were approached in these selected house, the next house were approached in case there was no women with child under 5 years. Before going to the field, Executive district officer (EDO) and Taulka health officer(THO) were informed and briefed about the study, permission was sought and letter was issued from directorate to the EDO and THO for the information and cooperation. A semi-structured questionnaire was developed and translated into local language , and were piloted and pre-tested before field implementation. Written consent was taken before staring the interview. Ethical approval was taken from ethical board of Health Services Academy Islamabad Pakistan. The data was analyzed through SPSS version 18.

\section{Results}

Two hundred respondents whose children were sick either because of ARI or Diarrhea during the preceding 15 days were interviewed; $181(90 \%)$ of these respondents were mothers. The mean age of the mothers was $28.25 \pm 4.7$ (SD). $96 \%$ of the mothers were housewives and only $4 \%$ were 
working lady, $13 \%$ and $5.5 \%$ were illiterate and doesn't have any formal education. $22 \%$ had attended the college and rest of the mothers had attended both Primary \& Secondary School. Regarding the children characteristics, $51 \%$ were infant with an evenly distributed gender. i.e. Male $=50.5 \%$ and Female $=49.5 \%$. (Table 1)

In table 2, breast feeding practices displays that shows $73.5 \%$ of mothers had started breast feeding immediate after birth, $67.5 \%$ were still breastfeeding at the time of interview, those who were not breastfeeding $13 \%$ of them fed up to 6 months, $8.5 \%$ fed up to 1 year, only $0.8 \%$ fed up to 2 Years and rest of mothers $(4.5 \%)$ didn't feed at all. those mothers who breast fed their children, among them $94 \%$ mothers were knowing that colostrums should be given at birth but only $85.5 \%$ mothers actually practice it on first day and rest did not. $25 \%$ mothers were against giving any prelacteal but the common prelacteal used was honey $(52 \%)$ by the rest of mothers. $5.5 \%$ of mothers doesn't what weaning is and $29 \%$ saying for start before 6months and rest of mothers said after 6months.

Table 1. Mother and Children's characteristics:

\begin{tabular}{|c|c|c|c|}
\hline \multicolumn{2}{|c|}{ Children Characteristics } & \multirow{2}{*}{$\frac{\mathrm{N}}{101}$} & \multirow{2}{*}{$\frac{(\%)}{50.5}$} \\
\hline Sor & Male & & \\
\hline $\mathrm{SUA}$ & Female & 99 & 49.5 \\
\hline \multirow{4}{*}{ Age (months) } & $<=6$ & 30 & 15.0 \\
\hline & $7-12$ & 72 & 36.0 \\
\hline & $13-18$ & 34 & 17.0 \\
\hline & $19-24$ & 64 & 32.0 \\
\hline \multicolumn{4}{|c|}{ Mother's Characteristics } \\
\hline \multirow{2}{*}{ Mother's occupation } & House wife & 192 & 96.0 \\
\hline & Working/Job & 8 & 4.0 \\
\hline \multirow{5}{*}{ Mother's education } & Illiterate & 26 & 13.0 \\
\hline & No formal education & 12 & 6.0 \\
\hline & Primary & 53 & 26.5 \\
\hline & High school & 65 & 32.5 \\
\hline & College/University & 44 & 22.0 \\
\hline
\end{tabular}

Table 2. Breast Feeding Practices:

\begin{tabular}{|c|c|c|c|}
\hline \multicolumn{4}{|c|}{ Breastfeeding Practices } \\
\hline \multirow{5}{*}{ How soon after birth should the newborn be given breast milk? } & Immediately & 147 & 73.5 \\
\hline & After one day & 25 & 12.5 \\
\hline & After two days & 14 & 7.0 \\
\hline & After three days & 4 & 2.0 \\
\hline & Other & 5 & 2.5 \\
\hline \multirow{2}{*}{ Are you still breastfeeding? } & Yes & 135 & 67.5 \\
\hline & No & 64 & 32.0 \\
\hline \multirow{2}{*}{ Should colostrums be given to the child? } & Yes & 189 & 94.5 \\
\hline & No & 11 & 5.5 \\
\hline \multirow{2}{*}{ If Yes, was colostrums given to the child? } & Yes & 171 & 85.5 \\
\hline & No & 29 & 14.5 \\
\hline \multirow{3}{*}{$\begin{array}{l}\text { Should anything else be given to the newborn other than breast } \\
\text { milk? }\end{array}$} & Yes & 125 & 62.5 \\
\hline & No & 51 & 25.5 \\
\hline & Don't know & 24 & 12.0 \\
\hline \multirow{3}{*}{ If, Yes what prelacteal was given to the child? } & Honey & 104 & 52.0 \\
\hline & Water & 3 & 1.5 \\
\hline & Others & 18 & 9.0 \\
\hline \multirow{3}{*}{ When should weaning be started? } & After 6 months of age & 131 & 65.5 \\
\hline & Before 6 months of age & 58 & 29.0 \\
\hline & Don't know & 11 & 5.5 \\
\hline
\end{tabular}


Table 3 shows that 180 out of $200(90 \%)$ mothers had been regularly taking their child for EPI vaccination and out of these mothers $94.5 \%$ administer Polio and $68 \%$ had administered Vit-A vaccination. Although $81 \%$ of mothers had confessed that they are regularly weighing their child but only $48.5 \%$ had growth monitoring card with them. Mean of the duration of illness was $3.85 \pm 2.252$ (SD) and range was 8 days.

Data was distributed into two further categories, Appropriate are those mothers who were taken their child to the registered medical professional (govt. or private) and
Inappropriate are those who sought the care other than that.

Table 4 reveals that $85.2 \%$ out of two hundred interviewed mothers had taken Appropriate Care whereas $14.8 \%$ mothers sought inappropriate care. Among these Appropriate Care sought mothers $69.4 \%$ had taken their children to Private Doctors and only $11.7 \%$ sought care from Government doctors. Within Inappropriate care sought mother 5\% waited for subsiding illness, $5.4 \%$ had taken their child to Pharmacist, $4.1 \%$ and $2.7 \%$ mothers had taken their child to traditional healer and Quake.

Table 3. History of Immunization:

\begin{tabular}{|c|c|c|c|}
\hline \multicolumn{4}{|l|}{ Immunization History } \\
\hline \multirow{2}{*}{ Was your youngest child weighed at birth? } & Yes & 162 & 81.0 \\
\hline & No & 38 & 19.0 \\
\hline \multirow{2}{*}{ Do you have a growth monitoring card for your youngest child? } & Yes & 97 & 48.5 \\
\hline & No & 103 & 51.5 \\
\hline \multirow{2}{*}{ Has your child been weighed in the last 4 months? } & Yes & 124 & 62.0 \\
\hline & No & 76 & 38.0 \\
\hline \multirow{2}{*}{ Do you regularly take your child for scheduled EPI vaccinations? } & Yes & 180 & 90.0 \\
\hline & No & 20 & 10.0 \\
\hline \multirow{2}{*}{ Do you have a vaccination card for your youngest child? } & Yes & 179 & 89.5 \\
\hline & No & 21 & 10.5 \\
\hline \multirow{2}{*}{ Do you give your child POLIO vaccine on National Immunization Days } & Yes & 189 & 94.5 \\
\hline & No & 11 & 5.5 \\
\hline \multirow{2}{*}{ Has your child been given Vitamin A in the past 6 months? } & Yes & 136 & 68.0 \\
\hline & No & 64 & 32.0 \\
\hline
\end{tabular}

Table 4. Patterns Care Sought:

\begin{tabular}{ccc}
\hline Type of Care Sought & $\%$ & \\
\hline Waited for illness to subside/no action taken & 5.0 & Inappropriate \\
\hline Was taken to private for medical care & 69.4 & Appropriate Care \\
\hline Was taken to government for medical care & 11.7 & Appropriate Care \\
\hline Purchased medicines from a pharmacy & 5.4 & Inappropriate \\
\hline Home treatment with traditional remedies & 4.1 & Appropriate Care \\
\hline Was taken to Quake & 2.7 & Inappropriate \\
\hline Was taken to temple/traditional healer & .9 & Inappropriate \\
\hline & 85.2 & Appropriate Care \\
\hline
\end{tabular}

Table 5. Awareness of Danger signs:

\begin{tabular}{cc}
\hline How would you recognized child's illness(awareness) & $\%$ \\
\hline Child develops fever & $27.0 \%$ \\
\hline Child becomes sicker & $24.0 \%$ \\
\hline Child is drinking poorly & $8.4 \%$ \\
\hline Child is not able to drink or breastfeed & $11.1 \%$ \\
\hline Child has fast breathing & $12.8 \%$ \\
\hline Child has difficulty in breathing & $11.1 \%$ \\
\hline Child has blood in stool & $1.7 \%$ \\
\hline Vomiting & $3.7 \%$ \\
\hline
\end{tabular}


Table 5 shows that Mother's awareness about danger signs of childhood illness was poor. None were aware of all danger signs and $27 \%$ were aware of fever, $24 \%$ knew about child getting sicker, $12.8 \%$ knew about the child's fast breathing, $11 \%$ knew that child has difficulty in breathing and is not able to breast feed respectively.

\section{Discussion}

It was interesting to note that most of the mothers sought appropriate care $(90.1 \%)$ and had taken their children to professional doctors and that a good proportion of the study population are literate $(81.5 \%)$ and well-off with better household income (>10000 Rs.). They are also privileged in that they had access to and could afford to seek appropriate care. Even then many of the mothers were still taking inappropriate care of their children, taking them to a pharmacy (5.4\%), quack or traditional healer $(2.7 \%$ \& $4.1 \%$ respectively). In the context of a good percentage of mothers taking appropriate care of their children, it was found that even though they do not recognize all the danger signs, they sought care when their child had even one of the danger signs. Mothers were aware of the dangers of a child getting more sick (24\%), having fever (27\%), fast breathing or is not able to breast feed $(12.8 \%)$. Only $5 \%$ waited at home for the illness to subside on its own possibly because of the high level of maternal literacy in the study area. A similar type of study done in western

Nepal by Chandrashekhar $T$ et al in 2006, found total family income, number of symptoms, mother's education and perceived severity of illness were the predictors of care seeking behavior and most of the mothers sought inappropriate care taking their child to a pharmacy $46.2 \%$ compared to appropriate care in $26.4 \%$. No care was sought for $8(2.7 \%)$ children and $26(8.9 \%)$ children received traditional or home remedies. 'Appropriate', 'prompt' and 'appropriate and prompt' care was sought by 77 (26.4\%), 166 $(56.8 \%)$ and $33(11.3 \%)$ mothers respectively. The mothers were aware of fever (51\%), child becoming sicker (45.2\%) and drinking poorly $(42.5 \%)$ as the danger signs of childhood illness. 11

The present study found that the mothers were more likely to seek care when they perceived illness, irrespective of how many danger signs they recognized.

When we compared our findings with those of other published studies, 14,15,16 we found both supporting and contrasting results. Pokher et al reported that gender of the child not only affect illness reporting but also affect the decision to choose a health care provider. However we did not find any such association in our study and mothers sought care for both male and female children equally15.

In context of the IMCI strategies to reduce mortality of children under five years by educating mothers and care givers on home care of children during illness and for recognizing signs of severe illness8, we assessed mother's knowledge and awareness regarding danger signs of childhood illness. We found that overall knowledge of mothers was poor, none the mothers were aware of all the danger sign despite the fact that $81.5 \%$ mothers were educated. Although mother generally could not recognize all the danger signs satisfactorily, a large proportion of them sought appropriate care for their children. Regarding the perceptions about pneumonia and diarrheal dehydration, although mothers perceive frequent stools, fever, cough and congestion, difficult and fast breathing as signs of illness, they rarely saw them as abnormal or dangerous; this may explains the few mothers who waited for illness to subside. In a study at Kintampos, Ghana, discovered barriers to care-seeking. They found that general signs of illness and symptoms are well recognized but some symptoms signaling danger signs were not spotted when they occurred. Although they recognize other symptoms, including frequent stools, fever, cough, difficult or fast breathing and restlessness, caregivers rarely see these as abnormal or dangerous. Poor recognition of danger signs is not the only barrier to care-seeking but also Caregivers do not seek medical care in half of the episodes they do not recognize as severe. 9 In contrast, in this study mothers sought care irrespective of the severity of illness. Previously many studies $11,12,13,14,16$ found that financial constraints and house hold income are major predictors for appropriate health seeking which is in agreement with our study, as we found that the proportion of mothers who had taken appropriate care were financially sound with a household income over Rs10,000.

In 2006, Shalley A. et al studied household practices to identify factors which really effect childhood morbidity and mortality in northern India; they found that delayed initiation of breast feeding (on 3rd day) was responsible for high childhood death in that area, although half of the mothers in their study recognized the danger signs.10In our study, breast feeding practices among the mothers were found satisfactory. $73.5 \%$ of mothers had started breast feeding immediate after birth and $85.5 \%$ of mothers had given clostrum to their child, $67.5 \%$ were still breast-feeding at the time of the interview, and $94 \%$ knew its importance. This can be the reason for not having a history of child deaths among the families of the subjects interviewed.

In the population of peri-urban India, the study of Amol $R$ Dongre et al showed that mothers gave first preference to private doctors and hospitals, explaining their availability and prompt care in an emergency, comparing them to public hospitals that are more distant and have lower quality of care;

However, these mothers also had poor knowledge of recognizing danger signs 17 . Our study also agreed with this as of the mothers who sought appropriate care, $69.4 \%$ had taken their child to private health care providers and only $11.7 \%$ to public care providers. The reasons given were the paucity of public health facilities versus a huge number of private clinics and hospitals, many with 24 hour emergency services in this urban study area. 


\section{Conclusions}

Study concluded that the Health seeking behviour of mothers has been found appropriate, however, their knowledge regarding the danger signs and illness of their children was reported low. It is recommended that some urgent awareness intervention by the policy makers for reducing the child death should be given to increase their knowledge on the danger sign of child diseases in Pakistan.

\section{REFERENCES}

[1] Preventable Diseases Continue to Kill Millions. 2009 [cited 2009 12th June]; Available from:http://www.oneworldhealth .org/global_burden

[2] WHO. Mortality - frequency and distribution; WHO Global burden of disease 2004 updated 2008. 2008 [cited 2009 15th June]; Available from: www.asiaing.com/the-global-burdenof-disease-2004-update.html

[3] Mankar MJ AM Mehendale, BS Garg. Role of Integrated Management of Childhood Illness (IMCI), Strategy for Changing Child Rearing Practices at Household and Community Level. Bombay Hospital Journal. 2008;50(4).

[4] NIPS. Pakistan demographic and health survey 2006-07. National Institute of Population Studies; 2008.

[5] Eshel N, et al. Responsive parenting: interventions and outcomes Bulletin of the World Health Organization. 2006; 84(12):992-9.

[6] Chandrasekhar T, et al. Care seeking behavior for childhood illness- a questionnaire survey in western Nepal. BMC International Health and Human Rights. 2006; 6(7).

[7] WHO. Pakistan acts to reduce child deaths from pneumonia: World Health Organization. http://www.who.int/inf-new/child2.htm Access on 29th June
2009

[8] WHO. Integrated management of childhood illnesses. [cited 2009 18th June]; Available from http://www.who.int/child_a dolescent_health/topics/prevention_care/child/imci/en/index. html

[9] Hill Z, et al. Recognizing childhood illnesses and their traditional explanations: exploring options for care-seeking interventions in the context of the IMCI strategy in rural Ghana'. Tropical Medicine and International Health 2003;8(7):668-76.

[10] Shally A, a TuhinaVerma,a and Monica Agarwal b. Danger signs of neonatal illnesses: perceptions of caregivers and health workers in northern India. Bulletin of the World Health Organization 2006; 84(8):819-26.

[11] Sreeramareddy CT, et al. care seeking behaviour for childhood illness- a questionair summary in western Nepal. BMC International Health and Human Rights. 2006; 6(23):6-7.

[12] Taffa N, Chepngeno G. Determinants of health care seeking for childhood illnesses in Nairobi slums. Trop Med Int Health. 2005; 10(3):240-5.

[13] Shaikh BT, and Juanita Hatcher. Health seeking behavior and health service utilization in Pakistan: challenging the policymakers. Journal of Public Health. 2004; 27:49-54.

[14] Pillai RK and WS, et al. Factors affecting decisions to seek treatment for sick children in Kerala, India. Social Science Medicine. 2003; 57(5):783-90.

[15] Pokhrel S and SR, et al. Gender role and child health care utilization in Nepal. Health Policy. 2005; 74(1):218-33.

[16] Pakhrel S and SR. Household decision making on child health care in developing countries: the case of Nepal. Health policy plan. 2004; 19(4):218-33.

[17] Amol RD, et al. A community based approach to improve health care seeking for newborn danger signs in rural wardha, India. Indian Journal of Pediatrics. 2008;76. 